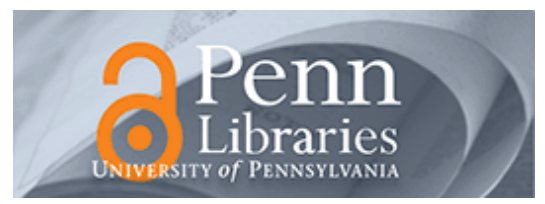

University of Pennsylvania

ScholarlyCommons

December 1985

\title{
Adaptive strategies for the control of natural motion
}

\author{
Daniel E. Koditschek \\ University of Pennsylvania, kod@seas.upenn.edu
}

Follow this and additional works at: https://repository.upenn.edu/ese_papers

\section{Recommended Citation}

Daniel E. Koditschek, "Adaptive strategies for the control of natural motion", . December 1985.

Copyright 1985 IEEE. Reprinted from Proceedings of the 24th IEEE Conference on Decision and Control, Volume 24, Part 1, 1985, pages 1405-1409.

This material is posted here with permission of the IEEE. Such permission of the IEEE does not in any way imply IEEE endorsement of any of the University of Pennsylvania's products or services. Internal or personal use of this material is permitted. However, permission to reprint/republish this material for advertising or promotional purposes or for creating new collective works for resale or redistribution must be obtained from the IEEE by writing to pubs-permissions@ieee.org. By choosing to view this document, you agree to all provisions of the copyright laws protecting it.

NOTE: At the time of publication, author Daniel Koditschek was affiliated with Yale University. Currently, he is a faculty member in the Department of Electrical and Systems Engineering at the University of Pennsylvania.

This paper is posted at ScholarlyCommons. https://repository.upenn.edu/ese_papers/396

For more information, please contact repository@pobox.upenn.edu. 


\title{
Adaptive strategies for the control of natural motion
}

\begin{abstract}
Earlier results of this author and others demonstrate that a broad range of robotic tasks can be commanded through relatively simple feedback controllers with a guarantee of global asymptotic stability. A weakness of such methods is the requirement that exact values of all dynamical parameters be available, since they are used to cancel the disturbance torques introduced by gravity. An adaptive strategy is reported here which guarantees stability and global boundedness of a natural controller in the absence of à priori information regarding dynamical parameters. The present results, however, are not yet satisfactory since they cannot assure convergence to the correct spatial position.

\section{Comments}

Copyright 1985 IEEE. Reprinted from Proceedings of the 24th IEEE Conference on Decision and Control, Volume 24, Part 1, 1985, pages 1405-1409.

This material is posted here with permission of the IEEE. Such permission of the IEEE does not in any way imply IEEE endorsement of any of the University of Pennsylvania's products or services. Internal or personal use of this material is permitted. However, permission to reprint/republish this material for advertising or promotional purposes or for creating new collective works for resale or redistribution must be obtained from the IEEE by writing to pubs-permissions@ieee.org. By choosing to view this document, you agree to all provisions of the copyright laws protecting it.

NOTE: At the time of publication, author Daniel Koditschek was affiliated with Yale University. Currently, he is a faculty member in the Department of Electrical and Systems Engineering at the University of Pennsylvania.
\end{abstract}




\title{
Adaptive Strategies for the Control of Natural Motion
}

\author{
Daniel E. Koditschek * \\ Center for Systems Science \\ Yale University, Department of Electrical Engineering
}

\begin{abstract}
Earlier results of this author and others demonstrate that a broad range of robotic tasks can be commanded through relatively simple feedback controllers with a guarantee of global asymptotic stability. A weakness of such methods is the requirement that exact values of all dynamical parameters be available, since they are used to cancel the disturbance torques introduced by gravity. An adaptive strategy is reported here which guarantees stability and global boundedness of a natural controller in the absence of $\grave{a}$ priori information regarding dynamical parameters. The present results, however, are not yet satisfactory since they cannot assure convergence to the correct spatial position.
\end{abstract}

\section{Introduction}

The term natural control refers to any control strategy which relies upon the unforced response of a time invariant closed loop system to achieve a desired motion. In the last few years, a number of researchers have begun to develop robot control methodologies based upon the use of pure feedback structures to encode a variety of robotic tasks - controlled impedances for performing mechanical work [1] and potential functions for obstacle avoidance $[2],[1]$ as well as trajectory shaping [3], [6], [7]. While the goals of these researchers are quite diverse and their work has been largely independent, a common theme has been a resort to the natural motion of the compensated plant to achieve some desired behavior.

A troubling flaw in the "ratural controllers" for which stability has been rigorously established [3], [8] is the reliance upon cancellation of the vector of gravitational torques. This would require exact knowledge of load and link dynamical parameters: while the latter may be assumed to be known more or less precisely from off line studies, the former can only be known, in general, in

\footnotetext{
* This work is supported in part by the National Science Foundation under grant no. DMC-8505160
}

the course of on-line manipulation and motion. In this paper, an adaptive technique is used to estimate and cancel the effect of gravity upon the robot arm. Since the unknown parameters enter linearly in the vector field, suitably modified sequential estimation algorithms from linear time invariant theory can be shown to achieve a stable and globally bounded closed loop system, however convergence to the desired end point cannot yet be guaranteed. Research attempting to improve these results is currently in progress.

\section{Notation and Preliminary Re- sults}

In the sequel we will refer to the standard rigid body model of robot arm dynamics,

$$
M(q) \ddot{q}+B(q, \dot{q}) \dot{q}+k(q)=\tau,
$$

where the generalized positions take values in joint space, $q \in J, B$ is linear in $\dot{q}$, and $M, B, k$ all vary in $q$ by polynomials of transcendental functions. Since $M$ is nonsingular for all robots of interest, this system is equivalent to

$$
\begin{aligned}
& \dot{x_{1}}=x_{2} \\
& \dot{x_{2}}=-M^{-1}\left(x_{1}\right)\left[B\left(x_{1}, x_{2}\right) x_{2}+k\left(x_{1}\right)-\tau\right]
\end{aligned}
$$

where the generalized positions and velocities take values in phase space, $x \triangleq\left[\begin{array}{l}q \\ \dot{q}\end{array}\right] \in \mathcal{P}$. We will occasionally refer to the output $\operatorname{map} r_{g}: J \rightarrow \mathcal{W}$, where $W \triangleq S O(3) \times \mathbf{R}^{3}$ is the workspace. The differential of a smooth function, $f$, or, (for our purposes) intechangeably, its "jacobian" matrix, will be denoted $d f$,

\subsection{Inverse Dynamics}

Given a reference trajectory, $q_{r}, \dot{q}_{r}, \ddot{q}_{r}$, the control strategs.

$$
\begin{aligned}
& \tau \triangleq\left[B-M K_{2}\right] \dot{q}+\left[k(q)-M K_{1} q\right]+r \\
& r \triangleq M\left[\ddot{q}_{r}+K_{2} \dot{q}_{r}+K_{1} q_{r}\right]
\end{aligned}
$$


$K_{1}, K_{2}>0$, may be shown to drive the errors,

$$
e \triangleq\left[\begin{array}{c}
q_{r} \\
\dot{q}_{r}
\end{array}\right]-x
$$

to zero. This methodology is recognizable in the robotics literature in a diversity of guises and is known most widely as the method of "computed torque" or "inverse dynamics".

Since it "linearizes" the equations of motion by exact cancellation of thousands of nonlinear terms from the rigid body model of robot dynamics (1), the question arises as to whether the method may be applied in practice at all: can the computation be effected quickly enough; can it be effected accurately enough? A number of researchers have persuasively argued that the answer to the first question is (or may soon be) yes [11],[12], which conclusion we will accept with no further discussion here. In the absence of reported empirical experience or analytical study, however, the second question remains open. Caution seems justified in light of (i) the inaccuracies in the rigid body model upon which such computation would be based; (ii) numerical inaccuracies in computation resulting from quantization effects; and (iii) uncertaintly regarding the values of the link dynamical parameters, and (in general) total ignorance of the load dynamical parameters. It seems well worth exploring control strategies which avoid exact cancellation as much as possible.

\subsection{Control of Natural Motion}

For a broad range of mechanical systems, the Hamiltonian is an exact expression for total energy. In a conservative force field this scalar function is a constant (defines a first integral of the equations of motion) and, in the presence of the proper dissipative terms, it must decay [13]. If the potential energy is "useful", the dissipation of total energy, will be associated with a natural motion which converges around a desired end point in $W[3]$. Strictly speaking, such motion satisfies a regulation criterion rather than the more general tracking or disturbance rejection criteria which are the hallmark of linear time invariant control theory. In exchange for this limitation, we gain the assurance of global stability without a complete reliance upon exact cancellation as in the previous section. Moreorer, there is good reason to believe that the transient natural response caused by such feedback regulators can effect motion in the large in a manner appropriate to a particular robotic task. The range of tasks for which a useful potential function may be found - i.e., one which shapes useful natural motion in the large - is the topic of extensive research by this author and others $[5],[2],[6]$, and will not be discussed further here. Instead, we concentrate upon the feedback controllers which achieve simple end point regulation in $J$.

These are based upon the joint space potential function,

$$
\varepsilon \triangleq \frac{1}{2}\left[q_{d}-q\right]^{\mathrm{T}} K_{1}\left[q_{d}-q\right]
$$

where $K_{1}$ is a positive definite symmetric matrix, and $q_{d} \in J$ is some desired end point of motion. The joint space regulator for (1) is based upon cancelling the gravitational field and matching the desired gradient with a dissipative term,

$$
\tau=k(q)-K_{2} \dot{q}-d \varepsilon^{T}
$$

( $K_{2}$ is also symmetric and positive definite), yielding the following result.

Theorem 1 (Takegaki and Arimoto [8], Koditschek (4)) The closed loop system of equation (2) under state feedback algorithm (4)

$$
\begin{aligned}
& \dot{x_{1}}=x_{2} \\
& \dot{x_{2}}=-M^{-1}\left[\left(B+K_{2}\right) x_{2}+K_{1}\left(q_{d}-x_{1}\right)\right]
\end{aligned}
$$

is globally asymptotically stable with respect to the equilibrium state $\left[\begin{array}{c}q_{d} \\ 0\end{array}\right] \in P$ for any positive definite symmetric matrices, $K_{1}, K_{2}$.

\section{Proof: Define the Lyapunov function,}

$$
v \triangleq \frac{1}{2}\left(x_{2}^{\mathrm{T}} M x_{2}+\left[q_{d}-x_{1}\right]^{\mathrm{T}} K_{1}\left[q_{d}-x_{1}\right]\right),
$$

whose time derivative is given by

$$
\dot{v}=\frac{1}{2} x_{2}^{\mathrm{T}} \dot{M} x_{2}-x_{2}^{\mathrm{T}}\left[\left(B+K_{2}\right) x_{2}+K_{1}\left(q_{d}-x_{1}\right)\right]+\left[q_{d}-x_{1}\right]^{\mathrm{T}} K_{1} x_{2} .
$$

It has been shown $[3]$ that $B=\frac{1}{2}[\dot{M}+J]$ where $J$ is skew-symmetric, hence we have

$$
\dot{v}=-x_{2}^{\mathrm{T}} K_{2} x_{2} \leq 0
$$

and it remains to demonstrate that $\left\{\left[\begin{array}{c}q_{d} \\ 0\end{array}\right]\right\}$ is the only positive invariant set in the subspace $\mathcal{L} \triangleq\{x \in$ $P: \dot{v}=0$ \}. The vector field on $\mathcal{L}$ is given by

$$
\left.f\right|_{\mathcal{L}}\left(x_{1}\right)=\left[\begin{array}{c}
0 \\
M^{-1} K_{1}\left(q_{d}-x_{1}\right)
\end{array}\right],
$$

while its tangent space is $T \mathcal{L}=\operatorname{Im}\left[\begin{array}{l}I \\ 0\end{array}\right]$. Thus $f_{\mathcal{L}} \notin T \mathcal{L}$, except at $\left[\begin{array}{c}q_{d} \\ 0\end{array}\right]$ (since $M^{-1} K_{1}$ is nonsingular), which is consequently the only positive invariant set in $\mathcal{L}$. The result follows according to LaSalle's invariance principle [9].

More interesting candidates for $\varepsilon$ are compositions of the kinematic map, $r_{g}(q)$ with a potential function on $W$, a construction affording automatic inverse kinematic solution and trajectory shaping in Cartesian coordinates as discussed in [5]. Unfortunately, in this case, trajectories may "stall" at a critical point of $r_{g}$ before reaching the desired spatial position. Research devoted to an exact characterization of the global attracting set for these more sophisticated regulators is currently in progress. It is important to add that a theoretically informed methodology for choosing the "damping" matrix, $K_{2}$, to insure nonoscillatory transients remains to be developed as well.

In contrast to the inverse dynamics procedure, a reliance upon natural motion obviates the need to cancel any portion of the robot dynamics beyond the "destabilizing" vector field due to gravity $-k(q)$. While $k(q)$ 
typically has a much simpler structure than the moment of inertia matrix, $M(q)$, or the coriolis and certripetal force matrix, $B(q, \dot{q})$, the procedure is still subject to the criticism raised previously. Of greatest concern, exact knowledge of the plant and load dynamical parameters would still be required to permit its implementation. We now attempt to dispense with this requirement.

\section{Adaptive Natural Control with Unknown Dynamical Parame- ters}

Theoretical understanding within the field of adaptive systems theory remains concentrated upon systems which are

- linear time invariant:

- of bounded dimension and known relative degree;

- minimum phase;

for these comprise what is in some sense the most general class of systems admitting adaptive control techniques whose stability may be guaranteed [14]. The standard formula for updating parameter estimates is based upon a sequential version of the ordinary least squares algorithm [15] and while its application (or a generalization) may often be apparent in settings which depart from these assumptions, its efficacy is not. In this section we will apply the standard updating algorithm to the robot equations (1) and indicate the problems that arise. While stability and global boundedness is assured, it will be seen that convergence properties are less than desirable, and that more research will be required to afford a useful result.

\subsection{A Special Nonlinear Adaptive Algo- rithm}

While little progress has been made in the adaptive control of general nonlinear systems, the robot equations (1) are sufficiently well structured that insights from the linear time invariant case are of considerable value. This intuition is most easily given formal expression by stating the following result.

Lemma 1 Consider the control system

$$
\dot{x}=a(x, p)+D(x, p)[H(x) p+u]
$$

where $a$ is a smooth vector field on the state space $X$, parametrized by a constant, $p \in \mathbf{R}^{q}$, with the property that for every value of $p$ in some known open subset $Q \subset \mathbf{R}^{q}$, $\dot{x}=a(x, p)$, has a globally asymptotically stable origin; and $D, H$ are smooth matrix valued functions of the appropriate dimension on $\chi$. Then for every $p \in \mathcal{Q}$, there exists a dynamical compensator:

$$
\dot{\dot{p}} \triangleq z(x, \hat{p}, p)
$$

such that under the feedback control

$$
u \triangleq-H \hat{p} .
$$

every solution of the closed loop system

$$
\begin{aligned}
& \dot{x}=a+D H[p-\hat{p}] \\
& \dot{\hat{p}}=z
\end{aligned}
$$

converges to a limit set in the subspace

$$
\mathcal{L} \triangleq\left\{\left[\begin{array}{l}
x \\
\hat{p}
\end{array}\right] \in \mathcal{L} \times \mathbf{R}^{q}: x=0\right\}
$$

Proof: $\quad$ Since $a(x, p)$ is globally asymptotically stable, for every $p \in \mathcal{O}$, there exists a positive definite Lyapunov Function, $v(x, p)$ with the property

$$
d v(x, p) \cdot a(x, p) \leq 0 .
$$

with equality only at the unique equilibrium state, $x=0$, for all $p \in \mathcal{Q}$, according to the converse Lyapunov theorems [10]. Define the adaptive law as

$$
-\dot{\hat{p}}=\dot{\tilde{p}} \triangleq-H^{\mathrm{T}} D^{\mathrm{T}} d v^{\mathrm{T}},
$$

where $\tilde{p} \triangleq p-\hat{p}$, and a Lyapunov candidate, for the closed loop system (6)

$$
w \triangleq v+\frac{1}{2} \tilde{p}^{\mathrm{T}} \tilde{p}
$$

We now have

$$
\dot{w}=d v \cdot a+d v \cdot D H \tilde{p}-\tilde{p}^{\mathrm{T}} H^{\mathrm{T}} D^{\mathrm{T}} d v^{\mathrm{T}}=d v \cdot a \leq 0
$$

with equality only on $\mathcal{L}$, which must therefore contain a globally attracting positive invariant set according to LaSalle's invariance principle [9].

If $p$ is interpreted as an unknown parameter vector appearing in a known vector field the lemma amounts to a nonlinear adaptive control procedure for the exact cancellation of a (presumably destabilizing) portion of the system dynamics. Of course, this interpretation is fatuous if $z$ depends explicitly upon $p$ : an adaptive adjustment law is completely impracticable if its computation requires the unknown parameters. In any case the result requires a very special nonlinear system whose fortuitous structure affords the strong assumptions implicit in the lemma's hypothesis, namely, that

- all state variables are available:

- the reference signal to be tracked is a constant (zero in this case);

- the vector field to be cancelled is known and linear in the unknown parameters.

Fortunately, the robot equations (1), in the context of a "regulation" problem fulfill these requirements.

\subsection{Adaptive Cancellation of Gravitational Torques}

In the appendix to this paper will be found a brief derivation of system (1) wherein it is shown that the vector of gravitational disturbance torques at each generalized position, $q$, may be written in the form

$$
k(q)=H(q) p .
$$


$H$, being a matrix which depends upon the jacobian of the kinematic transformation, is completely known and computable; $p$ is the constant vector of unknown dynamical parameters. We may now attempt to use the lemma developed above to cancel adaptively the destabilizing vector field,

$$
\left[\begin{array}{c}
0 \\
-M^{-1} H p
\end{array}\right]
$$

By setting the control input to be

$$
\tau \triangleq-u-K_{2} x_{2}-K_{1} x_{1}
$$

(with $x_{1} \triangleq q_{d}-q$ ) we will identify

$a(x, p) \triangleq\left[\begin{array}{c}x_{2} \\ -M^{-1}\left(x_{1}, p\right)\left[\left(B\left(x_{1}, x_{2}, p\right)+K_{2}\right) x_{2}+K_{1} x_{1}\right]\end{array}\right]$

and

$$
D(x, p) \triangleq\left[\begin{array}{c}
0 \\
-M^{-1}\left(x_{1}, p\right)
\end{array}\right] .
$$

According to Theorem 1, $a$ defines a globally asymptotically stable system as long as $M$ is non-singular, thus we identify

$$
\mathcal{Q} \triangleq\left\{p \in \mathrm{R}^{10 n}: \forall q \in J,|M(q, p)| \neq 0\right\},
$$

(where $n$ is the number of degrees of freedom), and simply assume that every real robot has a set of dynamical parameters, $p \in \mathcal{Q}$.

According to the lemma, the construction of the adaptive law requires use of a Lyapunov function for $a$. Unfortunately, the only presently available candidate is the total energy function,

$$
v \triangleq \frac{1}{2}\left[x_{2}^{\mathrm{T}} M x_{2}+x_{1}^{\mathrm{T}} K_{1} x_{1}\right]
$$

whose time derivative along trajectories of that system was shown to be negative semi-definite. Proceeding anyway, as in the proof of the lemma, we set

$$
u=-H\left(q_{d}-x_{1}\right) \hat{p}
$$

(recall, $x_{1}$ is now defined to be $q_{d}-q$ ) and the adaptive law as

$$
\begin{aligned}
\dot{\hat{p}} & \triangleq H^{\mathrm{T}}\left[0,-M^{-1}\right]\left[\begin{array}{c}
K_{1} x_{1}+d_{x_{1}} x_{2}^{\mathrm{T}} M x_{2}^{\mathrm{T}} \\
M x_{2}
\end{array}\right] \\
& =-H^{\mathrm{T}} x_{2} .
\end{aligned}
$$

Notice that this is a practicable procedure, since all explicit dependence upon $p$ is cancelled. The closed loop behavior is governed by the equation

$$
\begin{aligned}
\dot{x_{1}} & =x_{2} \\
\dot{x_{2}} & =-M^{-1}\left[\left(B+K_{2}\right) x_{2}+K_{1}\left(x_{1}\right)+H \tilde{p}\right] \\
\dot{\tilde{p}} & =H^{\mathrm{T}} x_{2} .
\end{aligned}
$$

Corollary 1 For all $q_{d} \in \mathcal{J}, \quad p \in \mathcal{Q}$, the closed loop adaptive robot system (8) has a stable origin and gives rise to bounded solutions whose limit set is contained in the subspace

$$
\mathcal{L} \triangleq\left\{\left[\begin{array}{l}
x \\
\tilde{p}
\end{array}\right] \in P \times R^{10 n}: x_{2}=0\right\}
$$

Proof: Define the positive definite Lyapunov candidate,

$$
v \triangleq \frac{1}{2}\left(x_{2}^{\mathrm{T}} M x_{2}+x_{1}^{\mathrm{T}} K_{1} x_{1}+\tilde{p}^{\mathrm{T}} \tilde{p}\right)
$$

whose time derivative is given by

$$
\begin{aligned}
\dot{v}= & x_{1}^{\mathrm{T}} K_{1} x_{2}-x_{2}^{\mathrm{T}}\left[\left(B+K_{2}\right) x_{2}+K_{1} x_{1}+H \tilde{p}\right] \\
& +x_{2}^{\mathrm{T}} \dot{M} x_{2}+\tilde{p}^{\mathrm{T}} H^{\mathrm{T}} x_{2} \\
= & -x_{2}^{\mathrm{T}} K_{2} x_{2} \leq 0 .
\end{aligned}
$$

The result follows according to LaSalle's Invariance principle [9].

Thus, each physical trajectory will converge to some spatial position $q_{0} \in J$, and the parameter estimate, $\hat{p}$, will converge to some constant $\hat{p}_{0} \in \mathbf{R}^{10 n}$. Unfortunately, the result says nothing about the relation of these constants to their desired values. In fact, the most likely result of this procedure would be entirely unsatisfactory. For all those positions $q_{d} \in J$ at which $H\left(q_{d}\right)$ has full rank, the origin of system (8) lies in the interior of a smooth submanifold of $\mathcal{L}$ specified by

$$
\mathcal{M} \triangleq\left\{\left[\begin{array}{c}
q \\
0 \\
\tilde{p}
\end{array}\right]: \tilde{p} \in H^{-1} K_{1}\left(q_{d}-q\right)\right\}
$$

which is a set of equilibrium states. Thus, not only is the origin non-attracting, but solutions will converge to constants in $\mathcal{M}$ however distant from the origin that manifold extends. Physically, this corresponds to a command torque based upon a spatial error whose corruption by the parameter error exactly balances the gravitational force vector at a particular point in $\mathcal{W}$.

While Theorem 1 guarantees the existence of alternative Lyapunov functions for $a$ possessed of a negative definite time derivative along that vector field, none have been explicitly constructed to date (to the best of this author's knowledge). Moreover, there is no guarantee that the adaptive vector field, $z$ resulting from such a construction will be found free of $p$ dependence. These questions are the subject of current research.

It is worth making one final remark about the technique considered here. Since all three nonlinear pieces, $M, B, k$, of the robot equations (1) are shown to be linear in $p$ in the appendix, it is intriguing to imagine an adaptive implementation of the inverse dynamics algorithm, which cancels all nonlinearities. Note, however, that $M^{-1}$, which appears in the phase space formulation (2) to which the lemma above might be applied is not linear in $p$.

\section{Conclusion}

It is proposed to cancel gravitational torque disturbances using an adaptive technique reminiscent of the standard linear time invariant procedures. Preliminary results, while guaranteeing global boundedness, are unsatisfactory since they cannot assure convergence to the correct end position. No other adaptive laws for robot control have been proposed in the literature (to the best of the author's knowledge) which achieve global stability, hence 
this approach would seem to deserve further investigation.

\section{A Lagrangian Formulation of Dy- namical Systems}

Here we show that the robot equations of motion are linear in the dynamical parameters. Let ' $p \triangleq\left[\begin{array}{c}\pi_{1} \\ \pi_{2} \\ \pi_{3} \\ 1\end{array}\right]$ be a point in the body of link $i$, expressed in, homogeneous coordinates, with respect to a frame, $R_{i}$, in that link. The matrix representation of this frame with respect to an inertial system in the robot base may be written as ${ }^{0} R_{i}$, and is a function of all joint variables more proximal than link $i$.

Since the velocity of the point in the base frame is given by ${ }^{0} \dot{p}_{i}={ }^{0} \dot{R}_{i}{ }^{i} p$ it follow's that the kinetic energy contributed by link $\mathrm{i}$ is given as

$$
\begin{aligned}
T_{i} & =\frac{1}{2} \int_{L_{i}}{ }^{0} \dot{p}_{i}{ }^{\mathrm{T}}{ }^{0} \dot{p}_{i} \rho\left(\pi_{1}, \pi_{2}, \pi_{3}\right) d \pi_{1} d \pi_{2} d \pi_{3} \\
& =\frac{1}{2} \int_{L_{i}} \operatorname{trace}\left\{{ }^{0} \dot{R}_{i}{ }^{i} p^{i} p^{\mathrm{T}}{ }^{0} \dot{R}_{i}{ }^{\mathrm{T}}\right\} \rho d \pi \\
& =\frac{1}{2} \operatorname{trace}\left\{{ }^{0} \dot{R}_{i} P_{i}{ }^{\circ} \dot{R}_{i}{ }^{\mathrm{T}}\right\}
\end{aligned}
$$

where $\bar{P}_{i}$ is the matrix of dynamical parameters for link i,

$$
P_{i} \triangleq\left[\begin{array}{ll}
\overline{M_{i}} & \overline{m_{i}} \\
\overline{m_{i}} & \overline{\mu_{i}}
\end{array}\right]
$$

with mass, $\overline{\mu_{i}}$, (three-space) centroid $\overline{m_{i}} / \overline{\mu_{i}}$, and moment of inertia $\overline{M_{i}}$. It follows, then that the total kinetic energy of the arm is given by

$$
\left.T=\sum_{i=1}^{n} \operatorname{trace}\left\{{ }^{0} \dot{R}_{i} P_{i}{ }^{0} \dot{R}_{i}{ }^{\top}\right\}=\sum_{i=1}^{n} \Pi_{\text {trace }}{ }^{0} \dot{R}_{i} \otimes{ }^{0} \dot{R}_{i}\right] p_{i}
$$

where $\Pi_{\text {trace }}$ is a projection onto the subspace of $R^{16}$ corresponding to the diagonal elements of its matrix representation, $\otimes$ is the Kronecker product, and $p_{i} \in \mathbf{R}^{16}$ is the vector formed by "stacking" each successive column of $P_{i}$ below the preceding one. Similarly, the potential energy, $U$ is given as a sum

$$
U \triangleq \sum_{i=1}^{n} \Pi_{3} R_{i} p_{i 4}
$$

where $\Pi_{3}$ is the projection onto the third coordinate of $\mathbf{R}^{4}$, and $p_{i 4}$ is the fourth column of $P_{i}$,

$$
p_{i 4} \triangleq\left[\begin{array}{l}
\overline{m_{i}} \\
\overline{\mu_{i}}
\end{array}\right] \text {. }
$$

Thus, the Lagrangian, $\mathcal{L} \triangleq T-U$ is linear in the dynamical parameters,

$$
p \triangleq\left[\begin{array}{c}
p_{1} \\
\vdots \\
p_{n}
\end{array}\right]
$$

and it follows immediately that the dynamical equations formed by

$$
\frac{d d_{\dot{q}} \mathcal{L}}{d t}-d_{q} \mathcal{L}=\tau^{T}
$$

are linear in those parameters as well.

\section{Acknowledgements}

I would like to thank Professor K.S. Narendra and Dr. A. Annaswamy at Yale for a number of very helpful discussions.

\section{References}

[1] N. Hogan, "Impedance Control: An Approach to Manipulation, Part I: Theory" ASME J. Dyn. Syst. (to appear) 1985

[2] O. Khatib, "Dynamic Control of Manipulators in Operational Space" Sixth IFTOMM Congress on Theory of Machines and Mechanisms, New Dehli, 1983 , p. 10

[3] D. E. Koditschek, "Natural Control of Robot Arms" Yale Center for Systems Science Technical Report No. 8409, Dec. 1984 (revised, Mar. 1985).

[4] D.E. Koditschek, "Natural Motion for Robot Arms" IEEE Proc. $23^{\text {rd }}$ CDC, Las Vegas, December, 1984 pp. $733-735$

[5] D. E. Koditschek, "Selection of Robot Natural Motion via Feedback" Proc. Fourth Yale Workshop on Applications of Adaptive Systems Theory, May, 1985.

[6] F. Miyazaki and S. Arimoto, "Sensory Feedback Based On the Artificial Potential for Robot Manipulators" Proc. 9th IFAC Budapest, Hungary, July, 1984.

[7] E. Saltzman and H. Scott Kelso, "Skilled Actions: A Task Dynamic Result" Technical Report SR-76, Haskins Lab, Yale University, 1983.

[8] M. Takegaki, and S. Arimoto, "A New Feedback Method for Dynamic Control of Manipulators" J. Dyn. Syst. Vol 102, pp.119-125, June, 1981.

[9] J. LaSalle and S. Lefschetz, Stability by Liapunov's Direct Method, Academic Press, N.Y., 1961.

[10] W. Hahn, The Stability of Motion, Springer-Verlag, NY, 1967.

[11] J. M. Hollerbach, "A Recursive Formulation of Manipulator Dynamics and a Comparative Study of Dynamics Formulation and Complexity", in Brady, et. al. (eds) Robot Motion, pp. 73-87, MIT Press, 1982.

[12] R. H. Lathrop, "Parallelism in Manipulator Dynamics", Int. J. Robotics Res. 4:2, pp. $80-102$, summer 1985.

[13] Sir W. Thompson and P. G. Tait, Treatise on Natural Philosophy, University of Cambridge Press, Cambridge, 1886 .

[14] K. S. Narendra and Y. H. Lin "Design of Stable Model Reference Adaptive Controllers", in Applications of Adaptive Control, Narendra and Monopoli (eds.), Academic Press, 1980.

[15] K. S. Narendra and L. S. Valavani, "Stable Adaptive Observers and Controllers" Proceedings of the IEEE vol. 64, no. 8, August, (1976) 